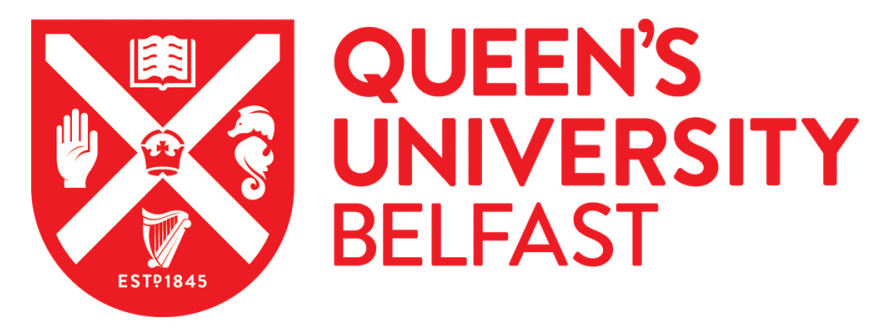

\title{
The use of eye-tracking to explore social difficulties in cognitively able students with autism spectrum disorder: A pilot study
}

Hanley, M., Riby, D. M., Carty, C., Melaugh McAteer, A., Kennedy, A., \& McPhillips, M. (2015). The use of eyetracking to explore social difficulties in cognitively able students with autism spectrum disorder: A pilot study. Autism, 19(7), 868-873. https://doi.org/10.1177/1362361315580767

Published in:

Autism

Document Version:

Peer reviewed version

Queen's University Belfast - Research Portal:

Link to publication record in Queen's University Belfast Research Portal

Publisher rights

(c) 2015 The Authors

\section{General rights}

Copyright for the publications made accessible via the Queen's University Belfast Research Portal is retained by the author(s) and / or other copyright owners and it is a condition of accessing these publications that users recognise and abide by the legal requirements associated with these rights.

Take down policy

The Research Portal is Queen's institutional repository that provides access to Queen's research output. Every effort has been made to ensure that content in the Research Portal does not infringe any person's rights, or applicable UK laws. If you discover content in the Research Portal that you believe breaches copyright or violates any law, please contact openaccess@qub.ac.uk. 
Brief Report

\section{The use of eye-tracking to explore social difficulties in cognitively able students with Autism Spectrum Disorder - a pilot investigation}

The majority of research on autism spectrum disorder (ASD) is focused on understanding its complex aetiology and behavioural impact, particularly in children (VanBergeijk, Klin, and Volkmar, 2008). However, it is important to be aware that the disorder can have significant impacts on the lives of even cognitively able adults (Howlin, Good, Hutton, and Rutter, 2004). Outcome for adults on the autism spectrum with average to high cognitive capacity is variable and social isolation plus secondary mental health problems can be significant (Howlin et al., 2004; Magiati, Tay and Howlin, 2014). Social impairments in ASD present significant barriers to social adaptation in adulthood (e.g. succeeding in education, employment and relationships; Eaves and Ho, 2008; Taylor and Seltzer, 2011; Shattuck et al., 2012). Given the rise in diagnoses, awareness and support for ASD in the 1990's, it is important to consider issues crucial to the daily living skills of adults. For example, the numbers of students with ASD in higher education is increasing, according to figures from support services in universities such as Cambridge (Ratcliffe, 2014) and Durham (Disability Support Durham University, personal communication, February 2015).

The transition to university life is significant for all students, both academically and socially. It is a transition where reliance on social cues is crucial for learning to navigate through an unfamiliar physical and social environment. Although intellectually comparable to their fellow students, students with ASD may be particularly vulnerable due to difficulties detecting and understanding social cues, which could impact on their progress. Although it is difficult to obtain figures for drop-out rates among students with an ASD, research indicates 
that students in higher education with 'hidden’ disabilities similar to ASD (developmental disorders such as Attention Deficit Hyperactivity Disorder) are more vulnerable and more likely to drop out of their courses than their typically developing peers (Schnepf, 2014; Wolf, 2011). Therefore, there is a timely need to explore the social deficits experienced by cognitively able adults with ASD attending higher education.

One challenge for researchers trying to probe social deficits in this group is that adults with ASD often deal with social situations/problems/tasks by relying more on cognitive reasoning skills and less on 'social abilities'. An example from the literature on mentalizing abilities in cognitively able ASD adults illustrates this using eye-tracking. Senju, Southgate, White, and Frith (2009) found that in typically developing (TD) adults, eye movements revealed anticipatory gaze in line with mentalizing. While watching a video version of a theory of mind task, TD participants spontaneously anticipated the behaviour of an actor, by looking at the window the actor would chose to reach through, based on his/her false belief. This was not the case for adults with ASD who, even though able to pass complex explicit theory of mind tasks, did not show anticipatory gaze indicative of mentalizing. This example illustrates one way in which individuals with ASD can apparently exhibit 'intact' social abilities, but achieve this in 'not-so-social' ways. It also highlights how eye-tracking is an ideal tool for capturing spontaneous social information processing in ASD while circumventing issues created by explicit task instructions and language demands typical of lab-based experiments.

Eye tracking research exploring atypical social attention in ASD has been informative , showing how social visual pursuit is atypical in ways that relate to core social difficulties (reduced gaze to eyes and poorer social functioning, Klin et al., 2002; Hanley et al., 2014; 
Brief Report

Jones et al., 2008; Riby \& Hancock, 2009). Recently, eye-tracking has been used during real face-to-face social interaction with children who have autism. Thus, Hanley et al. (2014) monitored gaze during a social interaction and explored links to social understanding in 17 primary school-aged children with autism compared to children with 14 language impairments and 16 TD children. Atypical gaze behaviour by children with autism (e.g. a lack of monitoring another person's facial expression in response to an unexpected event) was related to poorer mentalizing ability. Capturing the atypicality of social gaze in the precise situations with which people with ASD struggle has both theoretical and applied significance (e.g. see Chevalier, Kohl, Troiani, Brodkin, and Schultz, 2012).

In the current study, we focused on how real life social attention related to social functioning in adults with ASD. These were adults who were intellectually at a level that allowed them to study for a University degree and thus cognitively high functioning. Yet, they attended a University social group for support with the social side of University life. Therefore the aims of the study were to compare social attention profiles between university students with and without ASD during real social interaction. Importantly, real life eye tracking was used to advance our understanding of social attention characteristics during an interaction. A further aim was to explore how the attentional profile for students with ASD related to their social profile, as measured by the Social Responsiveness Scale (Constantino \& Gruber, 2005).

\section{Methods}

Participants 
Brief Report

Fifteen students with ASD were recruited through Disability Services at a UK University after responding to an email inviting students to participate in the study. Due to calibration difficulties, the final sample contained 11 individuals (20-46 years). All participants had an ASD diagnosis that was previously made by a clinician using DSM-IV criteria (APA, 1994). Severity of social deficits associated with autism was confirmed using the Social Responsiveness Scale (SRS; adult version completed by parents; Constantino and Gruber, 2005; Constantino and Todd, 2005). It was not possible to obtain SRS data for one female participant with ASD as it was not possible to contact her parents.

Students with ASD were matched to students with no known developmental or learning difficulties recruited through the same University. Matching was based on age, gender, verbal IQ and performance IQ (using Wechsler Abbreviated Scale of Intelligence; WASI; Wechsler, 1999; Table 1). The WASI provides a fast and reliable measure of IQ, containing four subtests, two of which measure verbal IQ (Vocabulary and Similarities) with a further two measuring non-verbal IQ (Block Design and Matrix Reasoning). It is designed for use with individuals in the age range of 6 to 89 years of age (Wechsler, 1999). The test took approximately 40 minutes to administer.

[Table 1]

Social responsiveness scale 
The SRS is a 65-item scale providing an overall social reciprocity score, and sub-domains for social awareness, social cognition, social communication, social motivation and autistic mannerisms. The total score and subscale scores are converted to T scores to determine if behaviour is within the 'normal' range (T score <60), the 'mild-moderate impairment' (6075) or shows 'severe impairments’ (76-90) that impact on daily functioning.

Although it was not possible to include a more comprehensive diagnostic evaluation such as the ADOS-2 (Lord et al., 2012) or the ADI-R (Lord et al., 1994) in the current study. The SRS has been widely used in research and is considered a reliable measure of autism symptomatology (e.g. see Speer et al. (2007) for use of the SRS in relation to eye tracking data and as diagnostic confirmation).

Design

The aim of this study was to capture spontaneous gaze behaviour during a real social interaction. However, making the participants aware that we were measuring where they were looking during the interaction was likely to make them very conscious of their gaze behaviour and subsequently to change their behaviour, thus undermining the central aim of the study. Therefore, we created a semi-structured interaction to elicit a social exchange without revealing the true aim. 
Participants were invited to complete a ‘colour perception’ eye tracking experiment. They sat in a chair 1.5 metres from a white wall. The SMI head-mounted eye-tracker sampled at $50 \mathrm{~Hz}$ and was a bicycle helmet with a mounted camera (SMI, Germany). Nine-point calibration involved recording participants' gaze while fixating points on the wall, corresponding to known points on the scene video. To validate accuracy of the calibration, we made a large board $(50 \times 60 \mathrm{~cm})$ containing different coloured circles at 9 locations. This also doubled as a prop for the 'colour perception' experiment.

When calibrated, participants were told the eye-tracker was not working and were asked to have a brief conversation with a student volunteer while the problem was fixed. The volunteer (an experimenter; 4 in total, 2 male, 2 female) sat on a stool against the blank wall and initiated a semi-structured 'conversation protocol' for approximately 3.5 minutes. The protocol involved three topics (eye-tracking problems; Christmas shopping; exam preparation) that were relevant to the time of year. It began with a rehearsed statement from the experimenter, followed by leading questions to which the participant could respond. Participants were then debriefed regarding the true purpose of the study. Participants were also asked follow up questions to probe whether they had awareness of the true purposes of the study or awareness that they had been deceived.

Awareness indicator

Participants were asked: 1) At any point did you think we were not doing a colour perception experiment? and 2) Did the conversation with the student feel natural/normal? Seven out of 
eleven TD and only one out of eleven ASD participants reported thinking we were not conducting a colour perception experiment. These figures were the same in terms of how the groups reported feeling the interaction was not natural. A Pearson chi square confirmed the group difference was statistically significant, $\chi^{2}(1, N=22)=7.071, p<.01$.

Video data coding and Reliability Analyses

Output videos from the eye-tracker with gaze cursor overlays were coded by two naïve raters, blind to participant diagnosis. Each video was coded on a frame-by-frame basis according to the position of the point of regard in relation to specified areas of interest (AOIs: eyes, mouth, face (including eyes, mouth), hair, body, wall, off-screen) using the Observer video coding package (Noldus, 1994) (See Figure 1 for example of video frame with point of regard). Fixations were defined as $\geq 3$ consecutive frames $(120 \mathrm{~ms})$ of stable gaze on an AOI, following the conventions of previous studies using head-mounted eye-tracking (Franchak, Kretch, Soska and Adolph, 2011). Rater 1 coded all videos, and Rater 2 coded 20\% of videos. Cohen's Kappa co-efficient and percentage of agreements were calculated using Observer to assess inter-rater reliability. Average $\kappa$ was 0.77 , and the average percentage of agreement was $82 \%$ indicating excellent agreement.

\section{Results}

Mixed factorial ANOVAs were used in the data analyses. A priori power calculations using G Power 3.0 (for the between, within and interaction effects) indicated that the sample size 
Brief Report

needed to detect a medium effect size, with a power of .8 and an alpha level of less than .05 , was 18 participants.

Gaze data

Figure 1 shows the percentage of fixations to AOIs across groups. A mixed measures ANOVA with factors Group (ASD, TD) and Region (face, body, wall) revealed a main effect of Region, $F(2,40)=126.271, p<.001, \eta^{2}=.86$, but no significant main effect of Group, $F(2,10)=.072, p=.791, \eta^{2}=.004$, nor an interaction, $F(2,40)=.250, p=.780, \eta^{2}=.012$. In terms of the different regions, planned Bonferroni comparisons revealed longer fixation to the face than body $(p<.001)$ or wall $(p<.001)$ regions, but body and wall regions were viewed for comparable time ( $p=.133)$. Both ASD and TD participants prioritised face information during the interaction.

Although both groups prioritised the face, analysis of the face region fixations revealed subtle but $t$ important group differences. A mixed measures ANOVA with factors Group (ASD, TD) and face Region (eyes, mouth) revealed a significant main effect of Region, $F(1,20)=25.223$, $p<.001, \eta^{2}=.47$, and a Group by Region interaction, $F(1,20)=7.778, p<.05, \eta^{2}=.15$, but a nonsignificant main effect of Group, $F(1,20)=1.934, p=.180, \eta^{2}=.09$. Overall, the eyes were viewed for longer than the mouth $(p<.001)$, but the TD group viewed the eyes for longer than the ASD group, $t(20)=2.462, p<.05, d=-1.049$, and the ASD group viewed the mouth for longer than the TD group, $t(20)=2.668, p<.05, d=1.137$. 
Brief Report

Participant characteristics and eye/mouth looking

As looking to the eyes and mouth differed between the groups, we explored correlations between time spent attending to these regions and individual differences. For all participants, neither age, VIQ nor PIQ were correlated with looking to the eyes (all $r$ 's $<.170$ and p's $>$.451). For ASD participants ( $\mathrm{N}=11)$, we explored correlations between the total score and domain scores from the SRS. The only significant relationships were between scores on the social awareness SRS domain and looking to the eyes and mouth. More looking at the eyes was associated with better social awareness $(\mathrm{r}=-.786, p<.01)$, and more looking at the mouth was associated with poorer social awareness $(\mathrm{r}=.791, p<.01)^{1}$.

\section{Discussion}

Using eye-tracking during social interaction, the current study compared the attention patterns of 11 adults with ASD to 11 TD adults, all of whom were University students. The aim was to explore the links between gaze behaviour and social deficits in cognitively able adults with ASD. This group is often overlooked in the literature as they are apparently ‘coping' and functioning well (e.g. managing to attend University). However, few adults with ASD 'grow-out' of autism-related difficulties. Understanding how these difficulties manifest in adulthood is important, particularly so that adults with ASD can be supported appropriately, be it in University or in employment for example. The current study provided

\footnotetext{
${ }^{1}$ Due to the small sample size, Spearman's Rho correlations were also conducted. The same relationships were found between social awareness and looking at the eyes $\left(r_{\mathrm{s}}=-.881, \mathrm{p}<.001\right)$, and looking at the mouth $\left(r_{\mathrm{s}}=\right.$ .826, $\mathrm{p}<.01)$.
} 
one example of these social interaction difficulties, showing how atypical attention to the eye and mouth regions was related to social awareness and an indirect measure of social understanding. At a gross level the gaze patterns of ASD adults appeared relatively 'typical' (e.g. spending similar amounts of time attending the face to the comparison group) but on closer inspection, attention to face regions was clearly different between ASD and TD adults.

Reduced attention to others' eyes and increased attention to mouths supports findings from social attention research in ASD using screen-based lab studies (Klin et al., 2002; Hanley et al., 2012; Riby \& Hancock, 2009). Importantly we show how this extends to real interaction (beyond screen viewing). Although a pilot study of this kind cannot be used to infer causal relationships we suggest that it demonstrates the usefulness of eye-tracking for probing some aspects of social information processing and understanding. It may be that reduced attention to eyes provides less access to subtle socio-communicative signals that are important for interpreting the dynamic of a social interaction. Using eye-tracking may help to pinpoint one way in which social interaction breaks down and therefore be helpful for devising management and / or training strategies for improving social skills in cognitively able adults with ASD. There is much scope for future work using this method and it goes without saying that replication with larger sample sizes is necessary. Future work may also include 'goldstandard' diagnostic measures such as the ADOS-2 (Lord et al., 2012) and the ADI-R (Lord et al., 1994) to explore in more detail the relationship between gaze behaviours and diagnostic profiles even in adults who are relatively high functioning.

\section{Conclusions}


This study emphasised that even when having a conversation with peers, cognitively able ASD adults who are studying at University may not follow the flow of an interaction or detect subtle nonverbal facial signals that are crucial to the interaction. Attending to the right information at the appropriate time is only one part of being successful socially but could have a knock on effect in terms of interpreting socio-communicative cues and using those cues to guide appropriate social behaviour. Here our results suggest a link between detecting social information and a measure of social awareness in these adults with ASD. While the scope of the current study was to explore social attention and social impairments with adults who are studying at University the findings are clearly relevant for adults with ASD more generally. For example, when dealing with any situations where adults with ASD are required to function socially and exchange information with others, such as employment situations (e.g. Taylor and Seltzer, 2011) or personal relationships during adulthood. It could be hypothesised that in such circumstances, those individuals who cannot detect subtle social signals appropriately may be those most at risk of managing the daily demands of social functioning. Further research should explore this issue to support adults with ASD.

\section{References}

American Psychiatric Association (1994) Diagnostic and statistical manual of mental disorders, DSM-IV (4th ed.). Washington, DC: Author. 
Brief Report

Chevalier C, Kohl G, Troiani V et al. (2012) The social motivation theory of autism. Trends in Cognitive Science, 16, 231-239.

Constantino, J. N. \& Gruber, C.P. (2005) Social Responsiveness Scale. Los Angeles, CA: Western Psychological Services.

Constantino, J.N. and Todd, R.D. (2005). Intergenerational transmission of subthreshold autistic traits in the general population. Biological Psychiatry, 57, 655-660.

Eaves LC and Ho HH (2008) Young adult outcome of autism spectrum disorders. Journal of Autism and Developmental Disorders, 38, 739-747.

Franchak JM, Kretch K S, Soska K C et al. (2011) Head-mounted eye-tracking: A new method to describe infant looking. Child Development, 82, 1738-1750

Hanley, M., McPhillips, M., Mulhern. G. \& Riby, D.M. (2013). Spontaneous attention to faces in Asperger Syndrome using ecologically valid static stimuli. Autism, 17(6): 754-761

Hanley M, Riby DM, McCormack T et al. (2014) Attention during social interaction in children with autism: Comparison to specific language impairment, typical development, and links to social cognition. Research in Autism Spectrum Disorders, 8, 908-924.

Happé F (1995) The role of age and verbal ability in the theory of mind task performance of subjects with autism. Child Development, 66, 843-855.

Howlin P, Goode S, Hutton J et al. (2004) Adult outcome for children with autism. Journal of Child Psychology and Psychiatry, 45, 212-229.

Jones, W., Carr, K. \& Klin, A. (2008). Absence of preferential looking to the eyes of approaching adults predicts level of social disability in 2-year-old toddlers with autism spectrum disorder. Archives of General Psychiatry, 65, 946-954. 
Klin A, Jones W, Schultz R, et al. (2002) Visual fixation patterns during viewing of naturalistic social situations as predictors of social competence in individuals with autism. Archives of General Psychiatry, 59(9), 809-816.

Lord C, Rutter M and Le Couteur A (1994) Autism Diagnostic Interview - Revised: A revised version of a diagnostic interview for caregivers of individuals with possible pervasive developmental disorders. Journal of Autism and Developmental Disorders, 24, 659-685.

Lord C, Rutter M, DiLavore PC et al. (2012) Autism Diagnostic Observation Schedule, Second Edition: ADOS-2. Western Psychological Services.

Magiati I, Tay XW and Howlin P (2014) Cognitive, language, social and behavioural outcomes in adults with autism spectrum disorders: A systematic review of longitudinal follow-up studies in adulthood. Clinical Psychology Review, 34, 73-86.

Ratcliffe R (2014) Helping students with asperger's syndrome prepare for university life. The Guardian. Retrieved from: http://www.theguardian.com/education/2014/sep/09/studentsaspergers-ready-university-life?commentpage=1

Riby, D.M., \& Hancock, P.J.B. (200a). Looking at movies and cartoons: eye tacking evidence from Williams syndrome and autism. Journal of Intellectual Disability Research, 53, 169-181 Senju A, Southgate V, White S et al. (2009) Mindblind eyes: An absence of spontaneous theory of mind in Asperger syndrome. Science, 325, 883-885.

Schnepf, S.V. (2014). Do Tertiary Dropout Students Really Not Succeed in European Labour Markets? IZA Discussion Paper No. 8015, March 2014. http://ftp.iza.org/dp8015.pdf

Shattuck PT, Narendorf SC, Cooper B et al. (2012) Postsecondary education and employment among youth with an autism spectrum disorder. Paediatrics, 129, 1042-1049. 
Brief Report

Speer LL, Cook AE, McMahon WM et al. (2007) Face processing in children with autism: Effect of stimulus contents and type. Autism, 11, 265-277.

Taylor JL and Seltzer M M (2011) Employment and post-secondary educational activities for young adults with autism spectrum disorders during the transition to adulthood. Journal of Autism and Developmental Disorders, 41(5), 566-574.

VanBergeijk E, Klin A and Volkmar F (2008) Supporting more able students on the autism spectrum: college and beyond. Journal of Autism and Developmental Disorders, 38, 13591370.

Wechsler D (1999) Wechsler Abbreviated Scales of Intelligence (WASI). USA: Psychological Corporation.

Wolf, L. E. (2011). College Students with “Hidden” Disabilities: The Freshman Survey Fall 2010. Higher Education Research Institute at

UCLA. http://www.heri.ucla.edu/PDFs/pubs/briefs/HERI_ResearchBrief_Disabilities_2011_ April_25v2.pdf 